\title{
Hipertensão do avental branco em adolescentes
}

\section{White coat hypertension in teenagers}

\author{
Bianca Fernandes Resende Candido ${ }^{1}$, Martha Elisa Ferreira de Almeida ${ }^{2}$
}

1. Bacharel em Nutrição pela Universidade Federal de Viçosa, Campus de Rio Paranaíba, Rio Paranaíba, MG. 2. Bacharel em Nutrição. Professora Adjunta da Universidade Federal de Viçosa, Campus de Rio Paranaíba, Rio Paranaíba, MG.

\section{Resumo}

objetivo: Avaliar a prevalência da hipertensão do avental branco em crianças e adolescentes, bem como os fatores de risco para a hipertensão arterial. Métodos: Analisaram-se alguns fatores de risco para a hipertensão arterial e os valores pressóricos com o avaliador utilizando vestimentas brancas e coloridas. Realizou-se o teste t pareado para avaliar os valores pressóricos dos adolescentes medidos utilizando diferentes vestimentas e a odds ratio para associar os fatores de risco e a hipertensão arterial, ambos a 5\%. Resultados: Participaram 115 crianças e 115 adolescentes, sendo que 27,90\% das neninas e 37\% dos meninos apresentaram excesso de peso; os perímetros da cintura e do pescoço estavam aumentados em 13,04 e 33,91\% dos avaliados, respectivamente. A hipertensão do avental branco foi identificada em $31,52 \%$ dos adolescentes, sendo que todos se apresentaram normotensos quando os valores pressóricos foram avaliados com roupas coloridas. Os fatores de risco associados à hipertensão arterial foram o excesso de peso, a ausência de aferição da pressão arterial e o perímetro do pescoço aumentado. Conclusão: Apenas os adolescentes sofreram interferência das vestimentas brancas e apresentaram a hipertensão do avental branco. Os avaliados apresentaram fatores de risco que podem contribuir para o desenvolvimento da hipertensão arterial.

Palavras-chave: Crianças. Adolescentes. Hipertensão do jaleco branco.

\begin{abstract}
Objective: To evaluate the prevalence of white coat hypertension in children and teenagers, as well as the risk factors for high blood pressure. Methods: Some risk factors for high blood pressure were analyzed, and the pressure values with the evaluator using white and colored garments. A paired t-test was held to evaluate blood pressure values of teenagers measured with the evaluators using different clothes and the odds ratio to associate risk factors and hypertension were both $5 \%$. Results: The participants were 115 children and 115 teenagers, with $27.90 \%$ of girls and $37 \%$ of boys were overweight; the waist and neck were enlarged by 13.04 and $33.91 \%$ of the individuals, respectively. The white coat hypertension was identified in $31.52 \%$ of teenagers, all of which showed up when normotensive blood pressure values were assessed in colorful clothes. Risk factors associated with arterial hypertension were overweight, lack of blood pressure measurement and the perimeter of the increased neck. Conclusion: Only teenagers experienced interference of white clothing and had a white coat hypertension. The reviews have risk factors that may contribute to the development of hypertension.
\end{abstract}

Keywords: Children. Teenagers. White coat hypertension.

\section{INTRODUÇÃO}

A Hipertensão Arterial (HA) pode ocorrer na infância e na adolescência em decorrência de diversos fatores, entre eles os antecedentes familiares ${ }^{1}$, a alimentação inadequada e o excesso de peso $^{2}$, os genéticos ${ }^{3}$, o etilismo, o tabagismo e o uso de hormônios do crescimento ${ }^{4}$. De acordo com dados da Sociedade Brasileira de Hipertensão ${ }^{5}$, na população brasileira a hipertensão arterial atinge cerca de $5 \%$ das crianças e adolescentes, $25 \%$ dos adultos e $50 \%$ daqueles com mais de 60 anos.

As características da hipertensão arterial com os valores pressóricos elevados constantemente vêm sendo alteradas com o passar dos anos, pois antes se tratava de uma patologia específica para adultos e idosos, e atualmente crianças e adolescentes vêm apresentando tal problema de saúde $^{6}$. A presença dos valores pressóricos elevados nesses estágios de vida pode culminar com diversas complicações cardiovasculares².

A Hipertensão do Avental Branco (HAB) é um dos fatores de risco para a hipertensão arterial sistêmica ${ }^{4}$. Ela é relatada como uma alteração na pressão arterial no momento da aferição em consultório ou por profissionais que utilizam o avental/jaleco branco, sendo que, ao aferi-la em ambientes como o domicílio ou por qualquer familiar, os valores pressóricos geralmente mantêm-se normais ${ }^{7-8}$. A hipertensão do avental branco pode acometer entre 20 e $40 \%$ dos indivíduos, sendo mais comum em mulheres e idosos ${ }^{7}$. Lurbe e Parati $^{9}$ identificaram que a hipertensão do avental branco estava presente em $18 \%$ das crianças e adolescentes na faixa etária entre 6 a 18 anos, sendo ressaltado o reduzido número de estudos nessa faixa etária, o que dificulta a obtenção de sua correta prevalência.

O objetivo deste estudo foi avaliar a prevalência da hipertensão do avental branco em crianças e adolescentes da rede pública de ensino da cidade de Rio Paranaíba - MG, bem como a presença dos fatores de risco para a hipertensão arterial.

\section{MÉTODOS}

Este é um estudo de caráter quantitativo, transversal e descritivo sobre as características sociodemográficas e econômicas,

Correspondência: Martha Elisa Ferreira de Almeida. Universidade Federal de Viçosa (UFV), Campus de Rio Paranaíba, Rio Paranaíba, Minas Gerais. Rodovia MG-230, Km 7. Rio Paranaíba - MG .CEP: 38.810-000.E-mail: martha.almeida@ufv.br

Conflito de interesse: Não há conflito de interesse por parte de qualquer um dos autores.

Recebido em: 13 Abr 2015; Revisado em: 14 Abr 2015; 7 Maio 2015; Aceito em: 26 Maio 2015. 
antropométricas e clínicas de crianças e adolescentes. Ele foi realizado após a aprovação do Comitê de Ética em Pesquisa da Universidade Federal de Viçosa, protocolo $n^{\circ} 508.233$, com crianças de uma Escola Municipal (Escola 1) e adolescentes de uma Escola Estadual (Escola 2), ambas de Rio Paranaíba - MG, que trouxeram assinados, nos dias das coleta de dados, os Termos de Consentimento e Assentimento Livre e Esclarecido.

Avaliou-se o perfil econômico por meio do Critério de Classificação Econômica Brasil ${ }^{10}$, e alguns fatores de risco para a hipertensão arterial.

A partir dos dados do peso e da altura, calculou-se o Índice de Massa Corporal (IMC), que foi analisado mediante os pontos de corte da Organização Mundial de Saúde ${ }^{11}$ por escore-z de acordo com a idade em meses, classificando os avaliados em magreza, eutrofia, sobrepeso, obesidade e obesidade grave. Os dados do perímetro da cintura e do pescoço foram considerados aumentados acima do percentil $90^{12}$.

A aferição da pressão arterial foi realizada com um equipamento semiautomático digital de braço (G-tech), por um único avaliador nutricionista devidamente treinado e capacitado para tal função. Primeiramente, ela foi feita no braço direito e, após alguns minutos, no braço esquerdo, sendo que, nos casos de divergência entre os valores, foi aferido novamente no braço direito. Após o cálculo da média entre os valores obtidos, tais dados foram comparados aos pontos de corte propostos pela V Diretriz da Sociedade Brasileira de Hipertensão ${ }^{13}$. Para avaliar a influência do avental branco nos valores pressóricos, primeiramente a pressão arterial foi aferida com a roupa do avaliador desprovida de coloração (branca). Nos indivíduos que apresentaram os valores pressóricos elevados, foram realizadas outras aferições, nas semanas subsequentes, utilizando vestimentas coloridas.

Utilizou-se o teste t pareado para comparar a diferença entre as aferições com vestimentas brancas ou coloridas, e a odds ratio para avaliar a associação dos fatores de risco e a hipertensão arterial, ambos a $5 \%$ de probabilidade.

\section{RESULTADOS}

Na escola 1, avaliou-se 56 meninas (48,69\%) e 59 meninos (51,30\%), e na escola 2 foram 64 meninas $(55,65 \%)$ e 51 meninos (44,35\%). A maioria das crianças foi classificada economicamente nas classes $\mathrm{C} 1$ e $\mathrm{C} 2$, enquanto na escola 2, a maioria dos adolescentes pertencia às classes B2 e C1. Apenas os adolescentes foram classificados economicamente na classe A.

A magreza foi identificada em $1,79 \%$ das meninas e $3,39 \%$ dos meninos da escola 1, e 3,13\% das adolescentes da escola 2 (FIGURA 1). A percentagem de alunos com sobrepeso foi maior na escola 1 , enquanto a de obesos predominou na escola 2 . Apenas entre as crianças (escola 1) foram identificados casos de obesidade grave.

Figura 1. Classificação do estado nutricional das crianças e adolescentes, segundo o parâmetro IMC/idade e o gênero.

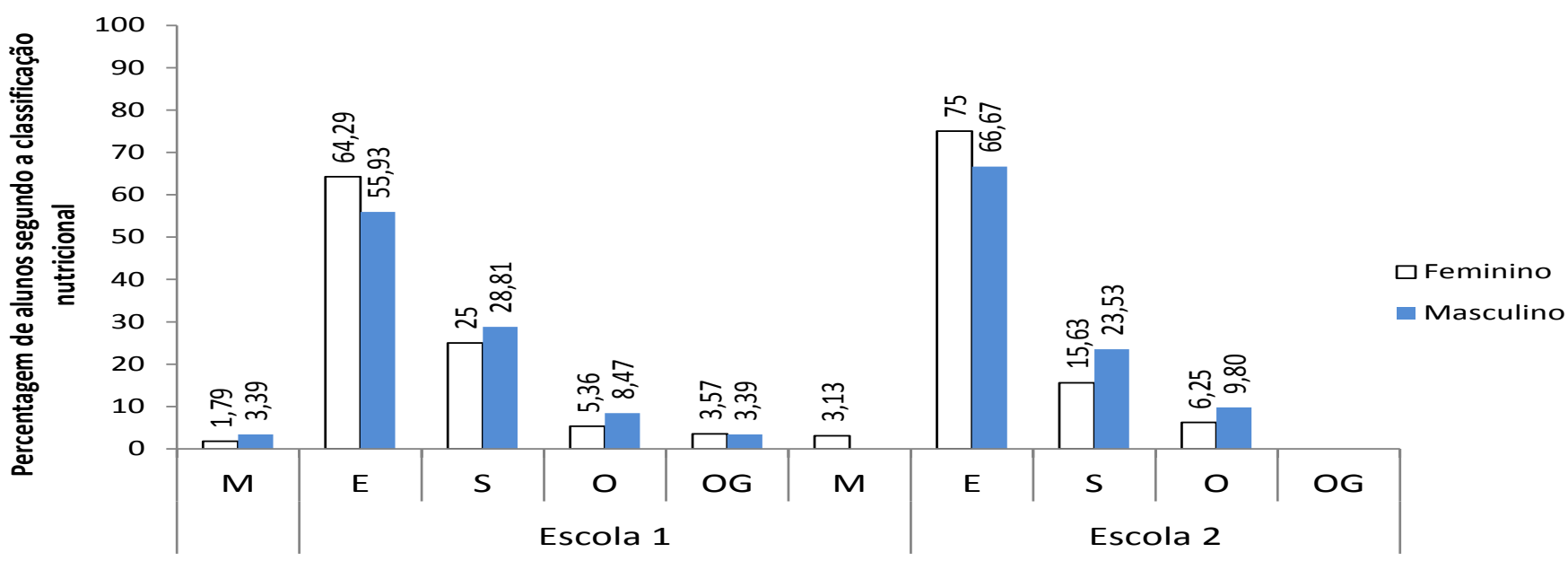

$\mathrm{M}=$ magreza, $\mathrm{E}=$ eutrofia, $\mathrm{S}=$ sobrepeso, $\mathrm{O}=$ obesidade, $\mathrm{OG}=$ obesidade grave

Ao utilizar vestimentas brancas, nenhuma das crianças apresentou valores pressóricos classificados como hipertensão arterial. Entretanto, 31,52\% ( $\mathrm{n}=35)$ dos adolescentes apresentaram hipertensão do avental branco, e nas outras avaliações (1 ou 2) utilizando vestimentas coloridas eles se apresentaram normotensos (FIGURA 2).

Houve diferença estatística $(p<0,05)$ dos valores pressóricos ao utilizar vestimentas brancas e coloridas (FIGURA 3), ressaltando a interferência da coloração no momento de avaliação da pressão arterial dos adolescentes.

Entre os fatores de risco avaliados, houve associação com a hipertensão arterial somente entre o excesso de peso $(p=$ $0,0065)$, a ausência da aferição pregressa da pressão arterial ( $p$ $=0,0128)$ e do perímetro do pescoço aumentado $(p=0,0036)$ (TABELA 1). Não houve associação do sedentarismo com a hipertensão arterial. 
Figura 2. Classificação dos valores pressóricos dos adolescentes, segundo o gênero, durante a aferição com vestimentas brancas e coloridas.

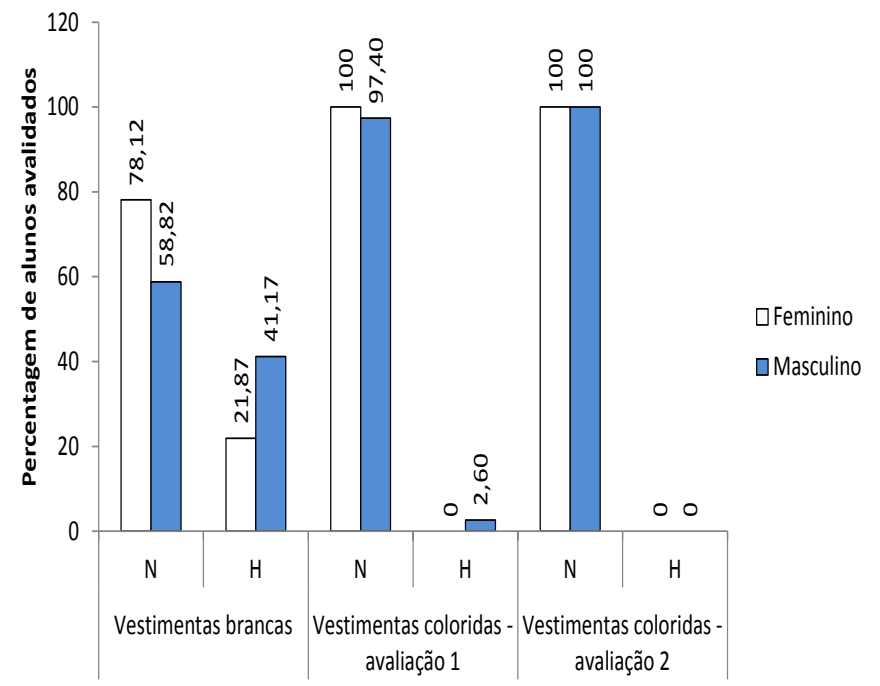

$\mathrm{N}=$ normotensão, $\mathrm{H}=$ hipertensão arterial, feminino $(\mathrm{n}=14)$, masculino $(\mathrm{n}=21)$
Figura 3. Valores pressóricos $(\mathrm{mmHg})$ dos adolescentes $(\mathrm{n}=115)$ utilizando vestimentas brancas e coloridas.

Tabela 1. Fatores de risco para a hipertensão arterial entre os alunos avaliados.

\begin{tabular}{|c|c|c|c|c|c|}
\hline Item avaliado & Hipertensão arterial & Normotensão & OR & IC 95\% & Valor de $p$ \\
\hline \multicolumn{6}{|c|}{ Classificação nutricional } \\
\hline Excesso de peso & $11(31,42 \%)$ & $63(32,20 \%)$ & 1 & & \\
\hline Eutrofia & $24(68,58 \%)$ & $127(65,12 \%)$ & 0,92 & $0,40-2,13$ & $0,0065^{*}$ \\
\hline \multicolumn{6}{|l|}{ Atividade física } \\
\hline Sedentário & $8(22,85 \%)$ & $30(15,38 \%)$ & 1 & & \\
\hline Ativo & $27(77,15 \%)$ & $165(84,61 \%)$ & 1,63 & $0,61-4,22$ & 0,3959 \\
\hline \multicolumn{6}{|c|}{ Hipertensão arterial familiar ${ }^{a}$} \\
\hline Sim & $13(37,15 \%)$ & $70(35,89 \%)$ & 1 & & \\
\hline Não & $22(62,85 \%)$ & $117(60,00 \%)$ & 0,99 & $0,44-2,21$ & 0,8746 \\
\hline \multicolumn{6}{|c|}{ Aferição pregressa da pressão arterial ${ }^{b}$} \\
\hline Não & $10(28,57 \%)$ & $102(52,30 \%)$ & 1 & & \\
\hline Sim & $25(71,43 \%)$ & $90(46,15 \%)$ & 0,35 & $0,15-0,82$ & $0,0128^{*}$ \\
\hline \multicolumn{6}{|c|}{ Perímetro do pescoço } \\
\hline Aumentado & $9(25,71 \%)$ & $69(35,38 \%)$ & 1 & & \\
\hline Normal & $26(74,29 \%)$ & $126(64,62 \%)$ & 0,29 & $0,12-0,70$ & $0,0036^{*}$ \\
\hline \multicolumn{6}{|l|}{ Perímetro da cintura } \\
\hline Aumentado & $4(11,43 \%)$ & $26(13,33 \%)$ & 1 & & \\
\hline Normal & $31(88,57 \%)$ & $169(86,67 \%)$ & 0,56 & $0,19-1,72$ & 0,3901 \\
\hline
\end{tabular}

Foi observado que 37,14\% (n=13) dos adolescentes com valores pressóricos elevados relataram a hipertensão arterial familiar nos avós, pai e mãe, fator que pode ter relação com a hipertensão arterial entre crianças e adolescentes.

Quando questionados se possuíam a pressão arterial elevada, $4,35 \%(n=5)$ das crianças e 4,35\% $(n=5)$ dos adolescentes afirmaram que sim, sendo que $41,74 \%(n=48)$ e $42,61 \%(n=49)$, respectivamente, não souberam relatar com precisão. A

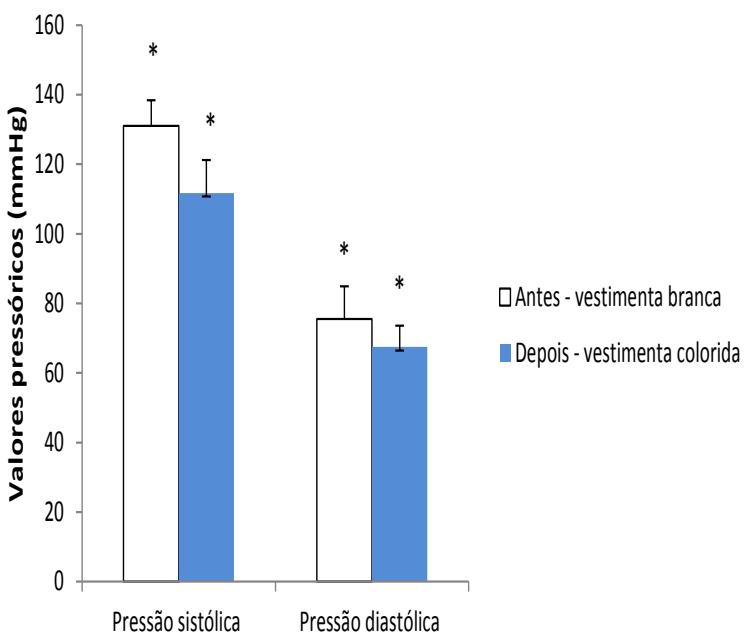

*Significativo pelo teste t pareado a 5\% de significância. ausência da aferição da pressão arterial foi citada por 52,30\% $(n=102)$ dos normotensos e $28,57 \%(n=10)$ dos hipertensos, sendo que os demais já haviam realizado tal procedimento no "hospital", "casa da avó" e na "escola".

O perímetro do pescoço aumentado foi identificado em 33,91\% $(n=78)$ dos alunos, sendo que entre aqueles com a hipertensão do avental branco a prevalência foi de $25,71 \%(n=9)$. O perímetro da cintura apresentou-se elevado em $13,04 \%(n=30)$ dos alunos 
avaliados, sendo que naqueles classificados com a hipertensão do avental branco a percentagem foi de $11,42 \%(n=4)$.

Quando analisado isoladamente os alunos com hipertensão do avental branco, constatou-se que $42,85 \%(n=15)$ faziam o consumo de álcool e 5,71\% (n=2) utilizavam cigarros ou outras drogas. $O$ uso de hormônios do crescimento foi relatado por $2,85 \%$ $(n=1)$ dos adolescentes e 5,71\% $(n=2)$ das adolescentes.

Entre os alunos avaliados, 46,09\% ( $n=106)$ passavam até duas horas por dia assistindo televisão e $87,83 \%$ ( $n=101)$ usando o computador. $O$ uso considerado excessivo (acima de 5 horas) da televisão foi relatado por $21,74 \%(n=50)$ e do computador por $15,65 \%(n=36)$ (FIGURA 4).

Figura 4. Horas disponibilizadas diante da televisão e do computador pelos avaliados nas duas escolas.

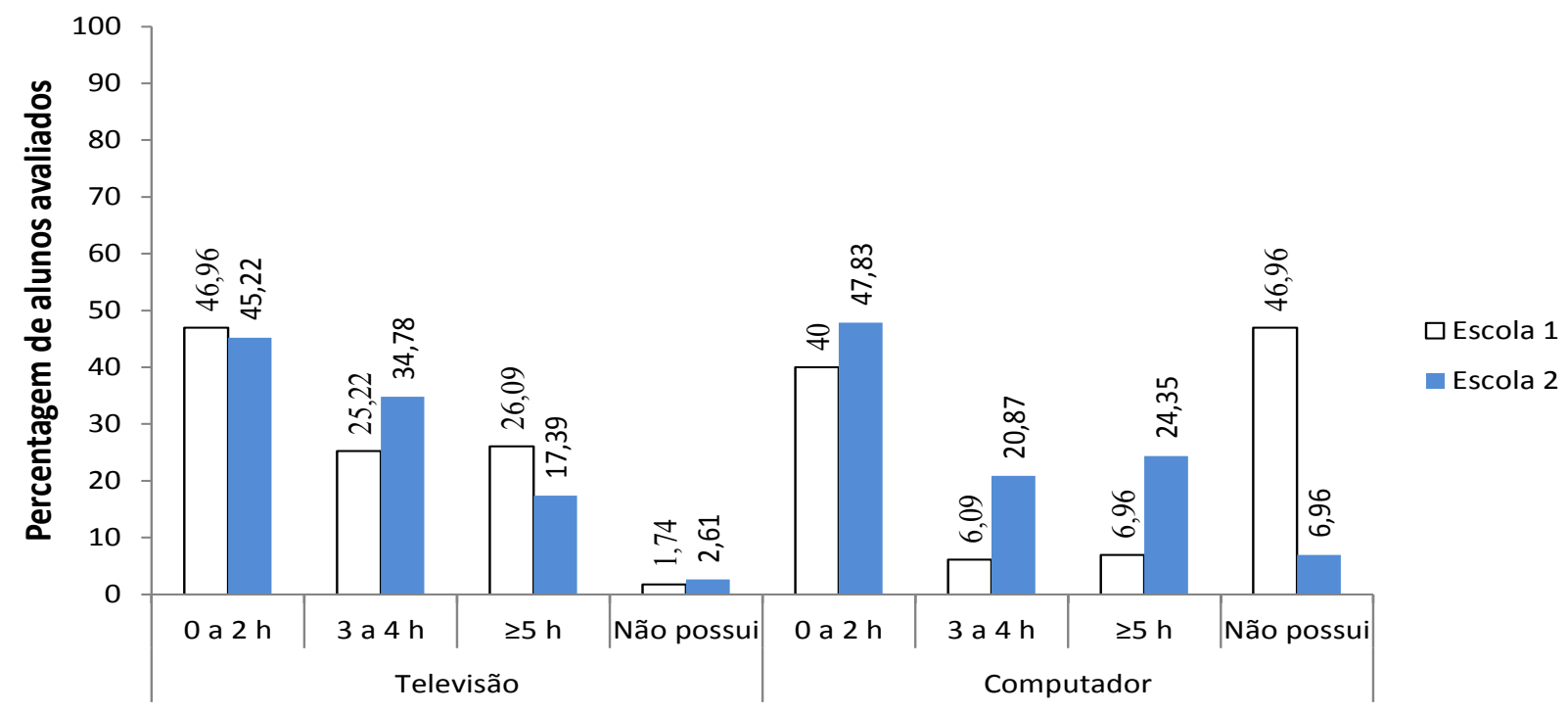

Entre os adolescentes, a ausência de posse da televisão foi relatada por 2,61\% ( $n=3)$, e $6,96 \%(n=8)$ não tinham o computador. Sugere-se que a classe econômica de algumas crianças pode estar contribuindo para a reduzida utilização do computador, pois $46,96 \%(n=54)$ relataram ausência de sua posse.

\section{DISCUSSÃO}

Como limitação do estudo destaca-se a repetição da aferição da pressão arterial somente naqueles indivíduos que apresentaram valores pressóricos aumentados após a mensuração pelo avaliador que utilizava vestimentas brancas. Sugere-se que, em outros estudos, haja a aferição dos valores pressóricos no mínimo duas vezes com a vestimenta da mesma cor, e que ocorra a inversão da sequência da cor das roupas.

A maioria dos avaliados pertencia às classes B e C. Essa classificação leva em consideração não apenas a renda, mas também a presença e o número de banheiros no domicílio, assim como o grau de escolaridade do chefe da família. A classe econômica a que o indivíduo pertence pode ser decisiva quanto à presença de fatores de risco para a hipertensão arterial, entre eles a elevada utilização de alimentos industrializados e prontos para o consumo, que tem sido justificado principalmente pela inserção das mulheres no mercado de trabalho, além do maior tempo destinado pelas crianças e adolescentes para o uso dos computadores e televisores ${ }^{14}$.
Quanto à magreza, tanto no gênero feminino quanto no masculino, a percentagem foi menor que $5 \%$, que é considerada aceitável para esta condição nutricional, segundo a Organização Mundial de Saúde ${ }^{15}$. Os dados obtidos foram inferiores aos de Pinto et al. ${ }^{16}$, em que a prevalência foi de $8,90 \%$ em ambos os gêneros, e superiores aos de Flores et al. ${ }^{17}$ que encontraram $2,50 \%$ das crianças e $1,65 \%$ dos adolescentes com baixo peso.

As crianças apresentaram mais sobrepeso e os adolescentes foram os mais obesos. Tais dados foram inferiores àqueles da Pesquisa de Orçamentos Familiares de 2008 e 2009, no qual $33 \%$ das crianças e adolescentes se encontravam com tal classificação nutricional ${ }^{18}$ e por Kaufmann e Ambernaz ${ }^{12}$ que identificaram $37,70 \%$ de excesso de peso. Entretanto, eles foram superiores aos achados de Schuch et al. ${ }^{19}$ que observaram uma prevalência de excesso de peso de $14,40 \%$ para as crianças e adolescentes e de Pinto et al. ${ }^{16}$ que o identificaram em $12,60 \%$ de crianças e adolescentes.

As meninas apresentaram menores prevalências de excesso de peso que os meninos, diferindo de Flores et al. ${ }^{17}$ que identificaram $27,60 \%$ nos meninos e $33,80 \%$ nas meninas. Somente entre as crianças, foi identificada a obesidade grave (3,57\% no gênero feminino e 3,39\% no masculino). 0 excesso de peso corporal é responsável por cerca de 20 a 30\% dos casos de hipertensão arterial, ${ }^{4}$ sendo que os riscos para desenvolver esta patologia aumentam em até seis vezes em meninos e três vezes nas meninas que possuem peso elevado, além de diminuir 
até $7 \%$ quando eles se apresentam eutróficos $20-21$.

Somente os adolescentes apresentaram a hipertensão do avental branco, sendo os dados semelhantes ao de Alves et al. ${ }^{22}$ que identificaram uma prevalência de $34,1 \%$ de hipertensos do avental branco e de Paiva et al. ${ }^{23}$ que encontraram 29\%, e superior ao resultado obtido por Lloret, Murakami e França ${ }^{8}$ que foi de $20 \%$. Neste estudo, foi observada uma maior prevalência da hipertensão do avental branco para os indivíduos do gênero masculino, assim como Mancia et al. ${ }^{24}$; entretanto, diferiu de Alves et al. ${ }^{22}$ e Paiva et al. ${ }^{23}$. A diferença dos valores pressóricos pode ocorrer devido à presença do avental branco (roupa típica dos profissionais de saúde) que é capaz de interferir no resultado final, ${ }^{25}$ sendo tal condição considerada um importante fator de risco para as mortes cardiovasculares ${ }^{24}$.

Houve interferência da coloração no momento da avaliação da pressão arterial dos adolescentes. Paiva et al. ${ }^{23}$ ressaltam que a medida da pressão arterial no consultório é de grande importância na identificação de alterações dos valores pressóricos, bem como da saúde do indivíduo. Entretanto, também é necessário realizar este procedimento em outros ambientes, uma vez que o diagnóstico de uma hipertensão do avental branco só é possível com variações no local de aferição. Silva e Farias Júnior ${ }^{26}$ destacam que apenas uma aferição da pressão arterial não é capaz de diagnosticar corretamente a hipertensão arterial, mas apenas prediz um risco de desenvolvêla, sendo necessárias outras aferições. Hansen et al. ${ }^{27}$ ao avaliarem mais de 7.000 pessoas identificaram que os indivíduos com hipertensão do avental branco eram mais propensos a desenvolver outros problemas cardiovasculares, bem como uma hipertensão arterial definitiva que é considerada uma condição não benigna.

As variações na quantidade de crianças e adolescentes com hipertensão arterial estão relacionadas com o tempo de acompanhamento do indivíduo, a forma e o número de aferições, sendo que, quanto mais avaliações, mais tranquila e relaxada a pessoa se torna com o procedimento, gerando o efeito de regressão média, em que as prevalências da hipertensão arterial que geralmente são da ordem de $13 \%$ nestas fases da vida, reduzem a valores próximos de 1 ou $2 \%^{20,28}$. Rinaldi et al. ${ }^{2}$ ao aferirem apenas uma vez a pressão arterial de crianças e adolescentes observaram que $9,6 \%$ foram consideradas com hipertensão arterial, e quando realizou-se mais duas aferições, essa percentagem reduziu-se para $3,2 \%$.

Entre os fatores de risco avaliados, houve associação com a hipertensão arterial somente entre o excesso de peso, a ausência da aferição pregressa da pressão arterial e do perímetro do pescoço aumentado. Silva et al. ${ }^{29}$ observaram que o sobrepeso e a obesidade em adolescentes estava diretamente relacionado ao desenvolvimento da hipertensão arterial.

Não houve associação da hipertensão familiar, do perímetro aumentado da cintura e do sedentarismo com a hipertensão arterial, assim como foi observado por Nascimento et al. ${ }^{21}$. Entretanto, Silva et al. ${ }^{29}$ observaram que a prática reduzida da atividade física estava diretamente relacionada com o desenvolvimento da hipertensão arterial. A inatividade física é um fator determinante para o excesso de peso, sendo que Silva et al. ${ }^{30}$ identificaram que o sedentarismo entre os adolescentes aumentou em $74 \%$ as chances de apresentarem excesso de peso, que é um dos fatores de risco para a hipertensão arterial. Foi observado que alguns adolescentes com valores pressóricos elevados relataram a hipertensão arterial familiar nos avós, pai e mãe, fator este que apresenta relação com a hipertensão arterial entre crianças e adolescentes ${ }^{4,20}$. Tal dado difere de Freitas et al. ${ }^{1}$ que observaram que $70,10 \%$ de escolares com casos familiares de hipertensão também eram hipertensos. Entretanto, o dado de Freitas et al. ${ }^{1}$ pode estar superestimado por uma única avaliação em triplicata dos valores pressóricos.

Os perímetros do pescoço e da cintura aumentados foram identificados em alguns alunos que apresentaram a hipertensão do avental branco. Tibana et al. ${ }^{31}$ observaram que os valores aumentados do perímetro do pescoço em mulheres estavam relacionados com o excesso de gordura corporal e, consequentemente, apresentavam um maior risco para o desenvolvimento das doenças cardiovasculares. Pinto et al. ${ }^{16}$ observaram que o perímetro da cintura estava aumentado em $8,70 \%$ dos avaliados e de Kaufmann e Ambernaz ${ }^{12}$ em $24 \%$. Este perímetro quando se encontra aumentado é preocupante, pois ele está diretamente relacionado com o excesso de peso, que é um fator predisponente para a hipertensão arterial ${ }^{12}$.

Entre os alunos com a hipertensão do avental branco, constatouse que alguns faziam o consumo de álcool e utilizavam cigarros ou outras drogas, bem como hormônios do crescimento. A presença destes fatores proporciona maiores chances de adquirir hipertensão arterial, e como esses indivíduos já são um grupo de risco com os valores elevados da pressão arterial, esses casos se tornam preocupantes ${ }^{4}$.

Alguns alunos deram o depoimento de que utilizavam de forma excessiva a televisão e o computador. A baixa prática de atividade física (lazer ativo) tem sido relacionada aos passatempos sem gasto energético como a televisão e o computador (lazer passivo) que podem culminar com o ganho de peso ${ }^{14}$. Spohr et al. ${ }^{32}$ relataram que, entre as crianças avaliadas, apenas $11,69 \%$ passavam mais de duas horas com esse tipo de entretenimento (televisão e computador), enquanto Silva et al. ${ }^{30}$ observaram que mais de $70 \%$ dos adolescentes destinavam mais de duas horas por dia para sua utilização, e Sarriera et al. ${ }^{33}$ identificaram que $24,89 \%$ dos adolescentes utilizavam aproximadamente 5,97 horas por dia para esses fins.

\section{CONCLUSÃO}

Apenas os adolescentes sofreram interferência da coloração das vestimentas do aferidor nos valores pressóricos, pois alguns deles foram classificados como hipertensos do avental branco. Os valores pressóricos na avaliação em que o avaliador utilizava vestimentas coloridas foram menores estatisticamente que na primeira avaliação, em que se utilizou as vestimentas brancas. Tanto as crianças quanto os adolescentes apresentaram alguns 
fatores de risco que podem contribuir para o desenvolvimento da hipertensão arterial definitiva, e consequentemente interferir de maneira negativa na qualidade de vida do indivíduo

\section{REFERÊNCIAS}

1. Freitas D, Rodrigues CS, Yagui CM, Carvalho RST, Marchi-Alves LM. Fatores de risco para hipertensão arterial entre estudantes do ensino médio. Acta Paul Enferm. 2012; 25(3):430-4. doi: http://dx.doi.org/10.1590/S010321002012000300017.

2. Rinaldi AE, Nogueira PCK, Riyuzo MC, Olbrich-Neto J, Gabriel GFCP, Macedo CS, et al. Prevalência de pressão arterial elevada em crianças e adolescente do ensino fundamental. Rev Paul Pediatr. 2012; 30(1):79-86. doi: http://dx.doi org/10.1590/S0103-05822012000100012.

3. Cesarino CB, Cipullo JP, Martin JFV, Ciorlia LA, Godoy MRP, Cordeiro JA et al. Prevalência e fatores sociodemográficos em hipertensos de São José do Rio Preto - SP. Arq Bras Cardiol. 2008 Jul; 91(1):31-5. doi: http://dx.doi. org/10.1590/S0066-782X2008001300005.

4. Sociedade Brasileira de Hipertensão. VI Diretrizes Brasileiras de Hipertensão. Arq Bras Cardiol. 2010; 95(1 supl. 1):1-51.

5. Sociedade Brasileira de Hipertensão. O que é hipertensão?[internet]. São Paulo: SBH; 2015 [Acesso 2015 Fev 15]. Disponível em: http://www.sbh.org. $\mathrm{br} /$ geral/geral.asp.

6. Ferreira JS, Aydos RD. Prevalência de hipertensão arterial em crianças e adolescentes obesos. Cienc Saude Colet. 2010 Jan; 15(1):97-104. doi: http:// dx.doi.org/10.1590/S1413-81232010000100015.

7. Guedis AG, Sousa BDB, Marques CF, Piedra DPS, Braga JCMS, Cardoso MLG, et al. Hipertensão do avental branco e sua importância de diagnóstico. Rev Bras Hipertens. 2008; 15(1):46-50.

8. Lloret RR, Murakami DY, França HH. Hipertensão do avental branco: conceituação, significado epidemiológico e clínico. Rev Fac Ciênc Méd Sorocaba. 2010; 12(3):1-3.

9. Lurbe E, Parati G. Out-of-office blood pressure measurement in children and adolescents. J Hypertens. 2008 Aug; 26(8):1536-9. doi: 10.1097/ HJH.0b013e32830a48e2. PubMed PMID: 18622226.

10. Associação Brasileira de Empresas de Pesquisa. Critério de classificação econômica Brasil. 2008

11. World Health Organization. The WHO child growth standards [internet]. [Acesso 2015 Maio 25]. Disponível em: http://www.who.int/growthref/en/.

12. Kaufmann CC, Albernaz EP. Prevalência e fatores associados ao excesso de peso em crianças de uma coorte no Sul do Brasil. Revista Ciência \& Saúde. 2013 Set-Dez; 6(3):172-80.

13. Sociedade Brasileira de Hipertensão. V Diretrizes Brasileiras de Hipertensão Arterial. Arq Bras Cardiol. 2007; 89(3):e24-e79.

14. Ribas AS, Silva LCS. Fatores de risco cardiovascular e fatores associados em escolares do município de Belém, Pará, Brasil. Cad Saúde Pública. 2014 JanMar; 30(3):577-86.

15. World Health Organization. The world health report 1995: bridging the gaps. Geneva: World Health Organization; 1995.

16. Pinto SL, Silva RCR, Priore SE, Assis AMO, Pinto EJ. Prevalência de préhipertensão e de hipertensão arterial e avaliação de fatores associados em crianças e adolescentes de escolas públicas de Salvador, Bahia, Brasil. Cad Saúde Pública. 2011 Jun; 27(6):1065-76.

17. Flores LS, Gaya AR, Petersen RDS, Gava A. Trends of underweight, overweight, and obesity in Brazilian children and adolescents. J Pediatr. 2013 Sep-Oct; 89(5):456-61. doi: 10.1016/j.jped.2013.02.021. PubMed PMID 23850108.
18. Instituto Brasileiro de Geografia e Estatística. Pesquisa de orçamentos familiares 2008-2009: antropometria e estado nutricional de crianças, adolescentes e adultos no Brasil [internet]. Rio de Janeiro: IBGE; 2010. [acesso 2015 Fev 15]. Disponível em: http://189.28.128.100/nutricao/docs/evento/ reuniao_nacional/2010/dia30/pesquisa_orcamento_familiar.pdf.

19. Schuch I, Castro TG, Vasconcelos FAG, Dutra CLC, Goldani MZ. Excesso de peso em crianças de pré-escolas: prevalência e fatores associados. J Pediatr. 2013 Mar-Abr; 89(2):179-88. doi: http://dx.doi.org/10.1016/j.jped.2013.03.003.

20. Araújo TL, Lopes MVO, Cavalcante TF, Guedes MG, Moreira PR, Chaves ES, et al. Análise de indicadores de risco para hipertensão arterial em crianças e adolescentes. Rev Esc Enferm USP. 2008 Mar; 42(1):120-6. doi: http://dx.doi. org/10.1590/S0080-62342008000100016.

21. Nascimento ESS, Ulbrich AZ, Panigas TF, Angarten VG, Carvalho T. Associação da hipertensão arterial sistêmica com fatores antropométricos e prática de atividade física em escolares. Rev Bras Ciên Saúde. 2013 Abr-Jun; 11(36):8-13. doi: $10.13037 /$ rbcs.vol11n36.1704.

22. Alves LMM, Nogueira MS, Godoy S, Hayashida M, Carnio EC. Prevalência de hipertensão do avental branco na atenção primária de saúde. Arq Bras Cardiol. 2007 Jul; 89(1):28-35. doi: http://dx.doi.org/10.1590/S0066$782 \times 2007001300006$.

23. Paiva AMG, Goulart MOF, Gomes MAM, Vasconcelos SML. Estudo clínico, nutricional e metabólico de portadores de hipertensão do avental branco e hipertensão mascarada e avaliação não invasiva da função vascular. Rev Bras Hipertens. 2010; 17(3):140-8.

24. Mancia G, Facchetti R, Bombelli M, Graci G, Sega R. Long-term risk of mortality associated with selective and combined elevation in office, home, and ambulatory blood pressure. Hypertens. 2006 May; 47:846-53. PubMed PMID: 16567588

25. Geleilete TJM, Coelho EB, Nobre F. Medida casual da pressão arterial. Rev Bras Hipertens. 2009; 16(2):118-22.

26. Silva KS, Farias Júnior JC. Fatores de risco associados à pressão arterial elevada em adolescentes. Rev Bras Med Esporte. 2007 Jul-Ago; 13(4):237-40. doi: http://dx.doi.org/10.1590/S1517-86922007000400005.

27. Hansen TW, Kikuya M, Thijs L, Björklund-Bodegard K, Kuznetsova T, Ohkubo $\mathrm{T}$, et al. Prognostic superiority of daytime ambulatory over conventional blood pressure in four populations: a meta-analysis of 7,030 individuals. J Hipertens. 2007 Aug; 25(8):1554-64. PubMed PMID 17620947.

28. Magalhães MEC, Brandão AA, Pozzan R, Brandão AP. Hipertensão arterial em crianças e adolescentes. Rev Bras Hipertens. 2002 Jul-Set; 9(3):2245-52.

29. Silva SL, Madrid B, Martins CM, Queiroz JL, Dutra MT, Silva FM. Influência de fatores antropométricos e atividade física na pressão arterial de adolescentes de Taguatinga, Distrito Federal, Brasil. Motri. 2013; 9(1):13-22. doi: 10.6063/ motricidade.9(1).2459.

30. Silva KS, Nahas MV, Hoefelmann LP, Lopes AS, Oliveira ES. Associações entre atividade física, índice de massa corporal e comportamentos sedentários em adolescentes. Rev Bras Epidemiol. 2008; 11(1):159-68.

31. Tibana RA, Teixeira TG, Farias DL, Silva AO, Marid B, Vieira A, et al. Relação da circunferência do pescoço com a força muscular relativa e os fatores de risco cardiovascular em mulheres sedentárias. Einstein. 2012 Jul-Set; 10(3):329-34. doi: http://dx.doi.org/10.1590/S1679-45082012000300013.

32. Spohr CF, Walker D, Azambuja CR, Farinha JB, Azevedo MR, Santos DL. Nível de atividade física de crianças do 1 을 ano do Ensino Fundamental. Rev Bras Ci e Mov. 2012; 20(4):106-11. 
33. Sarriera JC, Tatim DC, Coelho RPS, Bücker J. Uso do tempo livre por adolescentes de classe popular. Psicol: Reflex Crit. 2007; 20(3):361-7. doi: http://dx.doi.org/10.1590/S0102-79722007000300003.

Como citar este artigo/How to cite this article:

Candido BFR, Almeida MEF. Hipertensão do avental branco em adolescentes. J Health Biol Sci. 2015 Abr-Jun; 3(2):86-92.

J. Health Biol Sci. 2015; 3(2):86-92 